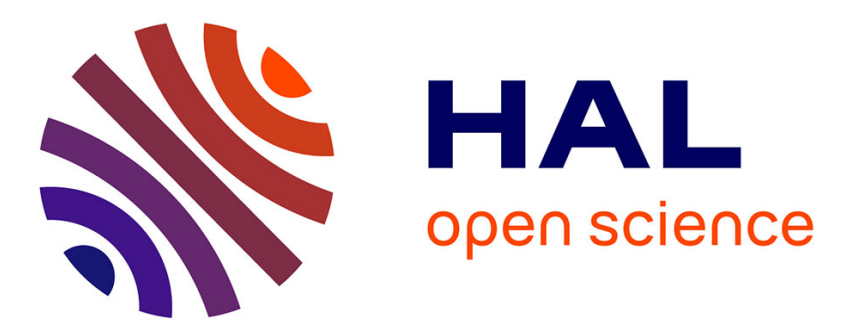

\title{
Étude par spectrométrie d'absorption infrarouge de l'interaction d'ions lourds avec un triacétate de cellulose
} J.P. Moliton, J.C. Vareille, J.L. Teyssier

\section{To cite this version:}

J.P. Moliton, J.C. Vareille, J.L. Teyssier. Étude par spectrométrie d'absorption infrarouge de l'interaction d'ions lourds avec un triacétate de cellulose. Revue de Physique Appliquée, 1974, 9 (4), pp.731-741. 10.1051/rphysap:0197400904073100 . jpa-00243840

\section{HAL Id: jpa-00243840 https://hal.science/jpa-00243840}

Submitted on 1 Jan 1974

HAL is a multi-disciplinary open access archive for the deposit and dissemination of scientific research documents, whether they are published or not. The documents may come from teaching and research institutions in France or abroad, or from public or private research centers.
L'archive ouverte pluridisciplinaire $\mathbf{H A L}$, est destinée au dépôt et à la diffusion de documents scientifiques de niveau recherche, publiés ou non, émanant des établissements d'enseignement et de recherche français ou étrangers, des laboratoires publics ou privés. 
Classification

Physics Abstracts

0.676

\title{
ÉTUDE PAR SPECTROMÉTRIE D'ABSORPTION INFRAROUGE DE L'INTERACTION D'IONS LOURDS AVEC UN TRIACÉTATE DE CELLULOSE
}

\author{
J. P. MOLITON, J. C. VAREILlE et J. L. TEYSSIER.
}

Laboratoire des Radiations Ionisantes

Université de Limoges, 123, rue Albert-Thomas, 87100 Limoges, France

(Reçu le ler février 1974, révisé le 28 février 1974)

\begin{abstract}
Résumé. - La spectrométrie d'absorption infrarouge constitue ici une technique d'investigation des dommages créés par l'irradiation d'un détecteur solide de traces. L'irradiation est obtenue par des ions $\mathrm{Kr}^{8+}(1 \mathrm{MeV} /$ nucléon$)$. Les détecteurs sont des films de triacétate de cellulose mis au point au laboratoire. Nous montrons que les trois types de liaison $\mathrm{C}-\mathrm{O}$ que contient la molécule de triacétate de cellulose sont affectés par l'irradiation pour des flux d'ions différents suivant le type de liaison et variant par pas de $2 \times 10^{12}$ ions $/ \mathrm{cm}^{2}$.
\end{abstract}

\begin{abstract}
Infrared Spectroscopy was used to study radiation damage effects on Solid State Track Detectors bombarded with $\mathrm{Kr}^{8+}$ ions ( $1 \mathrm{MeV} /$ nucleon). Cellulose triacetate films, produced by a method developed in our Laboratory were used. It is shown that the three kinds of $\mathrm{C}-\mathrm{O}$ bonds contained in the cellulose triacetate molecule are affected, according to their types, by different irradiation fluences, the latter varying by steps of $2 \times 10^{12} \mathrm{ions} / \mathrm{cm}^{2}$.
\end{abstract}

1. Introduction. - On sait que les dérivés cellulosiques, comme beaucoup de matériaux plastiques, constituent d'excellents détecteurs de traces de particules chargées [33]. Nous nous intéressons ici à l'un de ces détecteurs qui se présente sous la forme de films à base de triacétate de cellulose.

Cette étude a pour objet d'apporter quelques informations sur le mécanisme de l'interaction d'ions lourds (ions krypton de $1,1 \mathrm{MeV} /$ nucléon) avec le matériau détecteur. L'analyse fait appel à la spectrométrie d'absorption infrarouge et plus accessoirement à la spectrométrie $\mathrm{X}$.

2. Mode de fabrication des films. - Nous avons fabriqué nous-même des films à base de triacétate de cellulose pour pouvoir être maîtres le plus possible de leur composition et de leurs conditions d'obtention.

Nous réalisons d'abord un collodion à partir de flocons de triacétate de cellulose (qualité B. V.) de la Société Rhône-Poulenc: on dissout les flocons dans l'acide formique. Nous avons choisi cet acide parce que c'est un solvant relativement léger du triacétate, dont la température d'ébullition n'est pas trop élevée $\left(101^{\circ} \mathrm{C}\right)$ et surtout qui ne contient pas de traces de vapeur d'eau, contrairement à d'autres solvants légers.

Nous ajoutons ensuite un plastifiant. Il s'agit du diéthyle-phtalate qui a une densité $(1,230)$ voisine de l'acide formique $(1,220)$. Il a le mérite de ne pas rendre cassants les films.

Le mélange ainsi obtenu est agité en moyenne pendant vingt heures puis laissé au repos vingt-quatre heures: la solution atteint son propre équilibre; l'air piégé sous forme de bulles monte à la surface.

Le ballon qui contient le mélange au repos est muni d'un bec verseur qui permet d'étendre le plastique sur des glaces polies optiquement sans qu'il soit nécessaire de procéder à un transvasement qui provoquerait la formation de nouvelles bulles d'air (la qualité du poli des glaces conditionne en effet celle de la surface des films). Le ballon est monté sur un chariot mobile au-dessus des glaces; sa vitesse réglable permet de fixer l'épaisseur du dépôt.

Celui-ci sèche sur les glaces durant environ vingt heures dans une enceinte où l'on contrôle le renouvellement de l'air. Après quoi, on procède à un recuit à une température légèrement supérieure à $101^{\circ} \mathrm{C}$ de façon à éliminer les restes de solvant. Cette élimination est facilitée du fait que l'acide formique et le diéthyle-phtalate ont des densités voisines. Nous aurions préféré utiliser le tributyle phosphate au lieu du diéthyle-phtalate, car il améliore la tenue à l'eau du film ; malheureusement, il a une densité suffisamment faible $(0,97)$ par rapport à celle du solvant pour sécher surtout en surface et faire écran à la sortie des vapeurs d'acide formique lors $\mathrm{du}$ recuit. Il reste 
maintenant à décoller les films de leurs supports, ce qu'on obtient par trempage au bain marie à $50^{\circ} \mathrm{C}$.

Les caractéristiques des films sont les suivantes:

- Teneurs en plastifiant; le pourcentage en poids de plastifiant par rapport au triacétate est de $0 \%$, $5 \%, 10 \%, 15 \%$ et $20 \%$; les échantillons correspondants sont répertoriés $\mathrm{S}, \mathrm{P}^{5}, \mathrm{P}^{10}, \mathrm{P}^{15}$ et $\mathrm{P}^{20}$.

- Epaisseurs comprises entre 16 et $75 \mu \mathrm{m}$. Nous aurions pu fabriquer des films plus minces. Ils sont bien sûr plus fragiles mais surtout sans intérêt pratique compte tenu des conditions standard d'irradiation de nos échantillons (un ion krypton de $1 \mathrm{MeV} /$ nucléon a un parcours de $17 \mu \mathrm{m}$ ). Dans ce qui suit, l'épaisseur est portée en indice des lettres $S$ ou P.

Les deux faces des films ont un comportement identique vis-à-vis de l'enregistrement des traces individuelles d'ions krypton, contrairement à certains échantillons commerciaux de triacétate de cellulose [25].

3. Spectrographie infrarouge. - Nous avons enregistré les spectres d'absorption infrarouge d'échantillons vierges puis irradiés par des ions lourds. Notre objectif était double :

- Identifier avec le plus de certitude possible les bandes présentes.

- Evaluer les dommages crés par l'irradiation.

3.1 Conditions D'IRRAdiation. - Les irradiations ont été effectuées au cyclotron à énergie variable d'Orsay qui nous a fourni des ions krypton chargés huit fois, dont l'énergie est de $1,1 \mathrm{MeV} /$ nucléon.

Le nombre d'ions par $\mathrm{cm}^{2}$ est compris entre 1,77 et $9,25 \times 10^{12}$ selon les cas.

3.2 CONDITIONS D'ANALYSE DES SPECTRES. -3.2 .1$ Appareillage. - Nous avons utilisé :

entre 5000 et $200 \mathrm{~cm}^{-1}$ un spectrophotomètre Perkin Elmer 225,

entre 300 et $100 \mathrm{~cm}^{-1}$ un spectrophotomètre mis au point par Quintard [1].

Le film de triacétate est monté sur un support métallique et maintenu plaqué sur ce support de façon que les pertes par réflexion soient les mêmes d'un spectre à l'autre.

Nous aurions pu choisir une épaisseur voisine de celle du parcours des ions krypton, de l'ordre de $17 \mu \mathrm{m}$, de telle sorte que la totalité du matériau soit irradiée. Nous avons dû prendre une épaisseur de $45 \mu \mathrm{m}$ pour des raisons de tenue mécanique dans une étude des traces individuelles d'ions. Cependant on n'a pas intérêt à dépasser cette valeur, ne serait-ce que parce que l'absorption devient trop importante, au moins sur certaines bandes, et le récepteur associé au spectrophotomètre reçoit un faisceau d'intensité trop faible.

Les deux appareils délivrent une réponse qui permet d'obtenir le rapport $I_{1} / I_{2}, I_{1}$ et $I_{2}$ représentant respectivement les intensités d'une bande à son pied et à son maximum d'absorption.

3.2.2 Principe d'une analyse quantitative des spectres d'absorption. Nous nous sommes appuyés sur la loi de Beer. Après traversée d'un échantillon vierge d'épaisseur $b$, l'intensité $I_{0}$ du faisceau incident est réduite à $I$, intensité du faisceau transmis sur une fréquence donnée $v . I$ et $I_{0}$ sont liées par la relation :

$$
I=I_{0} \exp -\left(K_{v}+k_{v} \rho\right) b
$$

$K_{v}$ caractérise l'absorption correspondant au fond continu. Quant à $k_{v}$, c'est le coefficient d'absorption due aux groupements chromophores rapporté à l'unité de volume. Le nombre de ces groupements par unité de volume est $\rho$.

En règle générale, l'irradiation n'affecte qu'une tranche $a$ du film (Fig. 1) ; $a$ est de l'ordre de grandeur du parcours des ions lourds.

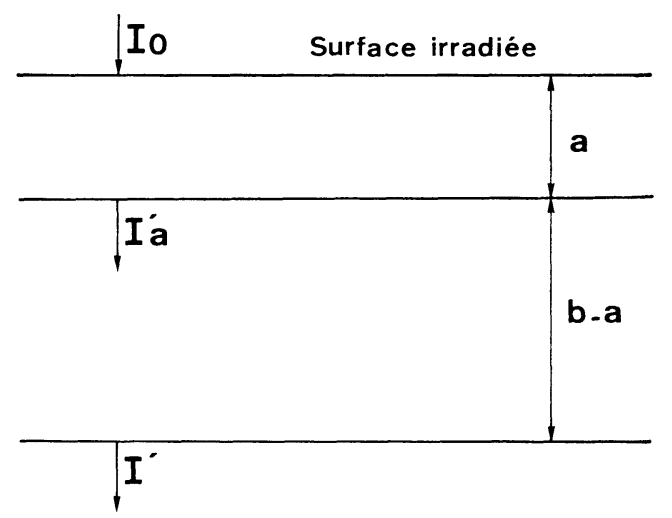

FIG. 1. - Schématisation de la zone irradiée du film.

L'intensité du faisceau transmis de fréquence $v$ est alors :

$I^{\prime}=I_{0} \exp -\left(K_{v}^{\prime} a+K_{v}(b-a)\right) \exp -k_{v} \rho^{\prime} b$

où $\rho^{\prime}$ est le nombre fictif des groupements chromophores par unité de volume après irradiation et supposés répartis dans tout le volume de l'échantillon. $K_{v}^{\prime}$ caractérise l'absorption relative au fond continu après irradiation. Soit en posant

$$
\begin{aligned}
& I_{0}^{\prime}=I_{0} \exp -\left(K_{v}^{\prime} a+K_{v}(b-a)\right) \\
& I^{\prime}=I_{0}^{\prime} \exp -k_{v} \rho^{\prime} b .
\end{aligned}
$$

Finalement :

$$
\frac{\rho^{\prime}}{\rho}=\frac{\log I^{\prime} / I_{0}^{\prime}}{\log I / I_{0}}=L
$$

$L$ est mesurable expérimentalement, et il en est de même de $\rho^{\prime} \mid \rho$.

En fait, ce que l'on cherche c'est non pas $\rho^{\prime}$ mais la densité volumique réelle $\bar{\rho}$ des groupements après irradiation. Pour trouver $\bar{\rho}$, on écrit les lois d'absorp- 
tion du faisceau dans les milieux d'épaisseur $a$ et $(b-a)$ pris cette fois-ci séparément :

$$
\begin{aligned}
& I_{a}^{\prime}=I_{0} \exp -\left(K_{v}^{\prime}+k_{v} \bar{\rho} a\right) \\
& I^{\prime}=I_{a}^{\prime} \exp -\left(K_{v}+k_{v} \rho\right)(b-a) .
\end{aligned}
$$

D'où :

$$
I^{\prime}=I_{0} \exp -\left(\left(K_{v}+k_{v} \rho\right)(b-a)+\left(K_{v}^{\prime}+k_{v} \bar{\rho}\right) a\right) .
$$

En comparant les expressions (1) et (2) de $I^{\prime}$ :

$$
\frac{\bar{\rho}}{\rho}=\frac{\rho^{\prime}}{\rho} \frac{b}{a}+1-\frac{b}{a}
$$

ou en fonction de la donnée expérimentale $L$

$$
\frac{\bar{\rho}}{\rho}=L \frac{b}{a}+\left(1-\frac{b}{a}\right) .
$$

4. Spectres d'absorption des composants. - En vue de faciliter l'identification des bandes présentes dans les spectres d'absorption des films, il a paru utile de tracer les spectres des différents constituants: acide formique, diéthyle-phtalate et flocons de triacétate.

Le spectre de l'acide formique (Fig. 2) placé dans une cuve dont les fenêtres sont en chlorure de sodium présente deux bandes nettement plus intenses que les autres :

$$
\begin{aligned}
& 1750 \mathrm{~cm}^{-1} v(\mathrm{C}=\mathrm{O}) \\
& 3200 \mathrm{~cm}^{-1} v(\mathrm{O}-\mathrm{H}) .
\end{aligned}
$$

En ce qui concerne le diéthyle phtalate (Fig. 3) dont le spectre est enregistré, à l'aide d'une cuve munie de fenêtres en chlorure d'argent, il faut signaler l'existence de trois bandes que nous retrouverons plus loin :

$740 \mathrm{~cm}^{-1}: \delta(\mathrm{C}-\mathrm{H})$ en dehors du plan benzénique d'après [2].

$$
\left.\begin{array}{l}
1580 \mathrm{~cm}^{-1} \\
1600 \mathrm{~cm}^{-1}
\end{array}\right\} \begin{aligned}
& \text { bandes propres au noyau benzé- } \\
& \text { nique [2]. }
\end{aligned}
$$

Pour les flocons de triacétate, on a dû fabriquer une pastille contenant $100 \mathrm{mg}$ de triacétate pour $1 \mathrm{~g}$ de $\mathrm{KBr}$. Compte tenu de la bonne résolution du spectrophotomètre nous avons constaté la présence de deux bandes à $948 \mathrm{~cm}^{-1}$ et $872 \mathrm{~cm}^{-1}$, dont l'existence n'a pas été mentionnée jusqu'à présent, du moins à notre connaissance (Fig. 4). Nous les attribuons au cycle pyrannosique de la cellulose par comparaison avec ce que l'on sait sur le $\beta$-D-glucopyrannose, la cellobiose et la cellulose (tableau I) [3-4]

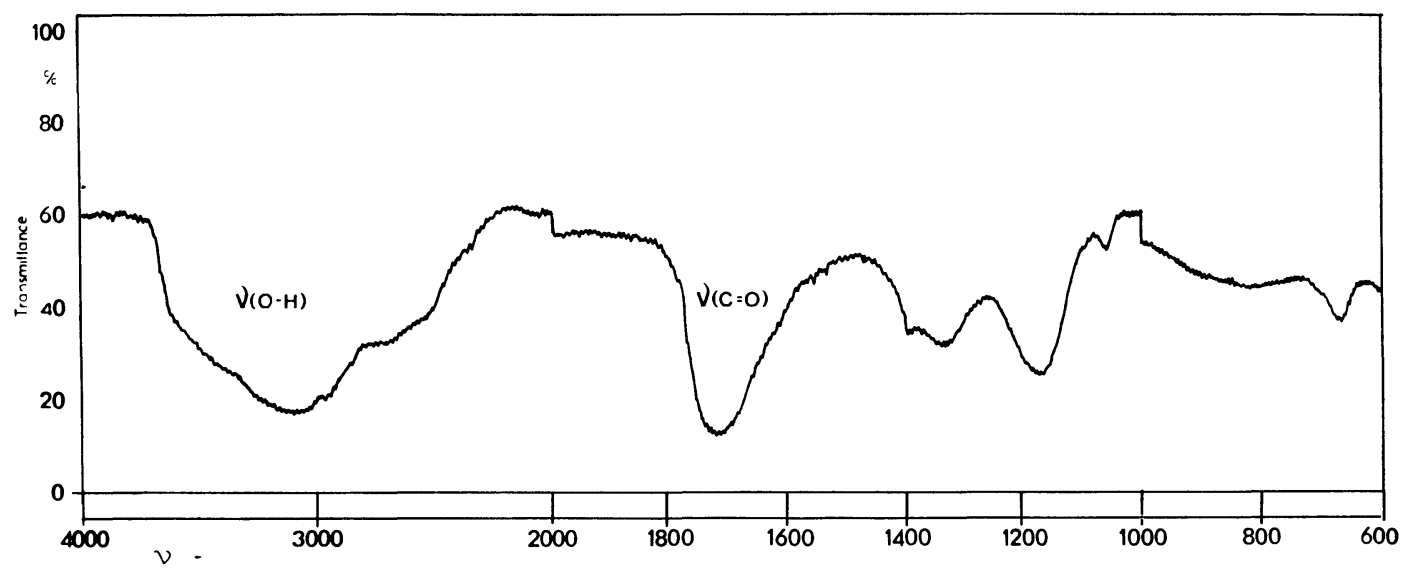

FIG. 2. - Spectre d'absorption de l'acide formique.

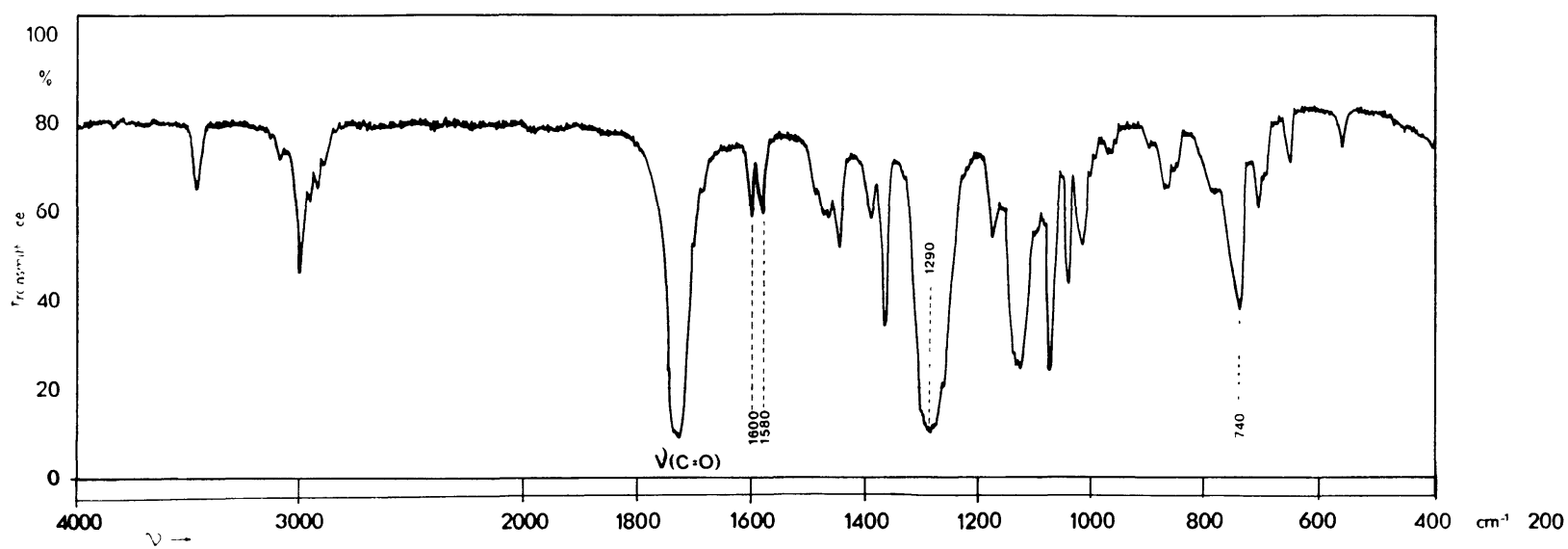

Fig. 3. - Spectre d'absorption du diéthyle-phtalate. 


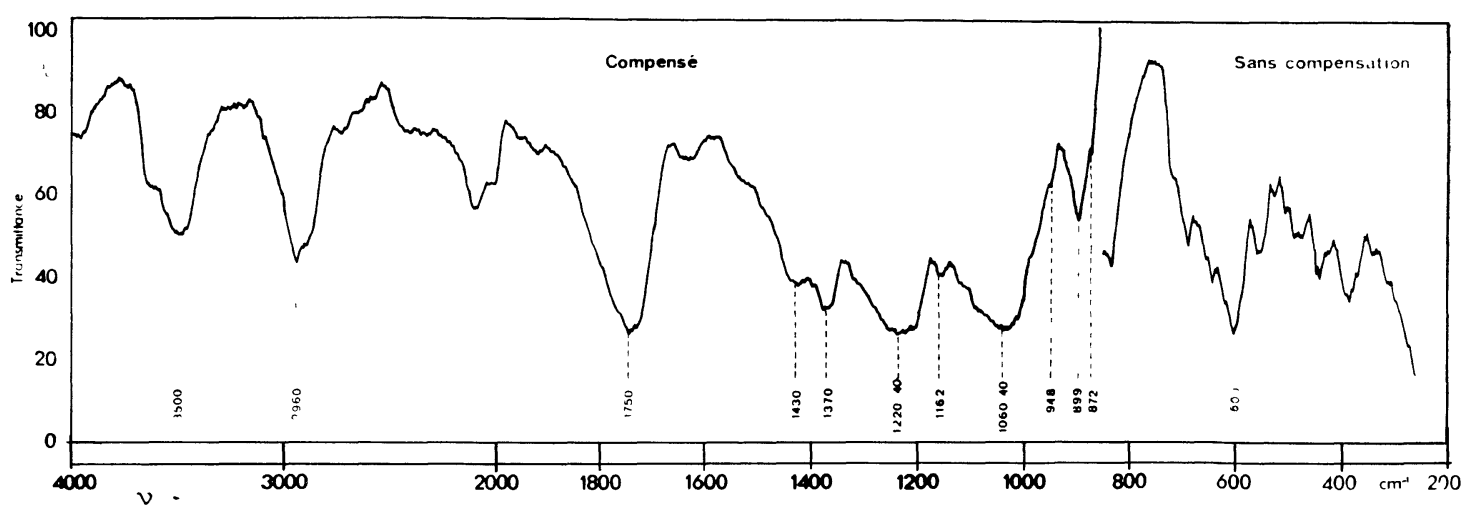

FIG. 4. - Spectre d'absorption des flocons de triacétate de cellulose.

Les autres bandes (tableau II) ont été identifiées avec certitude, sauf en ce qui concerne la région comprise entre 1000 et $1200 \mathrm{~cm}^{-1}$. Trois hypothèses ont été formulées à son sujet, tout particulièrement en ce qui concerne la bande à $1162 \mathrm{~cm}^{-1}$.

Il peut s'agir :

- du cycle pyrannosique [5-6-7],

- d'une vibration d'allongement $v(\mathrm{C}-\mathrm{O})$ ou d'une déformation $\delta(\mathrm{O}-\mathrm{H})$ [8],

- d'une vibration d'allongement antisymétrique du pont $\mathrm{C}_{(4)}-\mathrm{O}_{(6)}-\mathrm{C}_{(7)}$ [9-10].

\section{TABLEAU I}

Les bandes du cycle pyrannosique dans les polymères à base de $\beta$-D-glucopyrannose.

$\begin{array}{lcc} & \text { Type 1 } & \text { Type 2 } \\ \beta \text {-D-Glucopyrannose } & 909 \mathrm{~cm}^{-1}[3] & 896 \mathrm{~cm}^{-1}[3] \\ \beta \text {-Cellobiose } & 925 \mathrm{~cm}^{-1}[3] & 892 \mathrm{~cm}^{-1}[3] \\ \text { Cellulose } & 914 \text { et } 933 \mathrm{~cm}^{-1}[3] & 894 \mathrm{~cm}^{-1}[3] \\ \text { T. A. C. } & 948 \mathrm{~cm}^{-1} & 872 \mathrm{~cm}^{-1}\end{array}$

\section{TABLEAU II}

Identification des bandes du spectre d'absorption dans le triacétate de cellulose

Nombre d'onde $\mathrm{cm}^{-1}$

$3490-3500$

2960

1750

1430

1370

1320

$1220-1240$

$1162(1120,1050,1036)$

948

899

872

$835 \quad 490$

$690 \quad 470$

$640 \quad 445$

$600 \quad 437$

$550 \quad 390$

$525 \quad 395$
Attribution

Références

$v(\mathrm{O}-\mathrm{H})$

dans tous les cotons et dérivés cellulosiques

$v(\mathrm{C}-\mathrm{H})$

$v(\mathrm{C}=\mathrm{O})$

du radical acétyle

$\delta\left(\mathrm{CH}_{2}\right)$

balancement des hydrogènes du radical acé-

tyle ; bande couplée avec celle à $899 \mathrm{~cm}^{-1}$

$\delta\left(\mathrm{C}-\mathrm{CH}_{3}\right)$

du radical acétyle

$\delta\left(\mathrm{CH}_{2}\right)$ ou $\delta(\mathrm{OH})$

$v(\mathrm{C}-\mathrm{O})$

du radical acétyle

$\mathrm{C}_{(11)}-\mathrm{O}_{(9)}-\mathrm{C}_{(7)}-\mathrm{O}_{(6)}-\mathrm{C}_{(4)}$

Type 1 des sucres

$\delta\left(\mathrm{CH}_{2}\right)$

rotation plane des hydrogènes des carbo-

[2-7-15-16]

[6-7-16-18]

[16]

[6-7-16-18]

nes (6) ; bande couplée avec celle à $1430 \mathrm{~cm}^{-1}$

Type 2 des sucres

Non identifiées 
Les atomes de carbone et d'oxygène sont repérés dans la molécule de cellulose selon la notation de Meyer et Misch (Fig. 5).

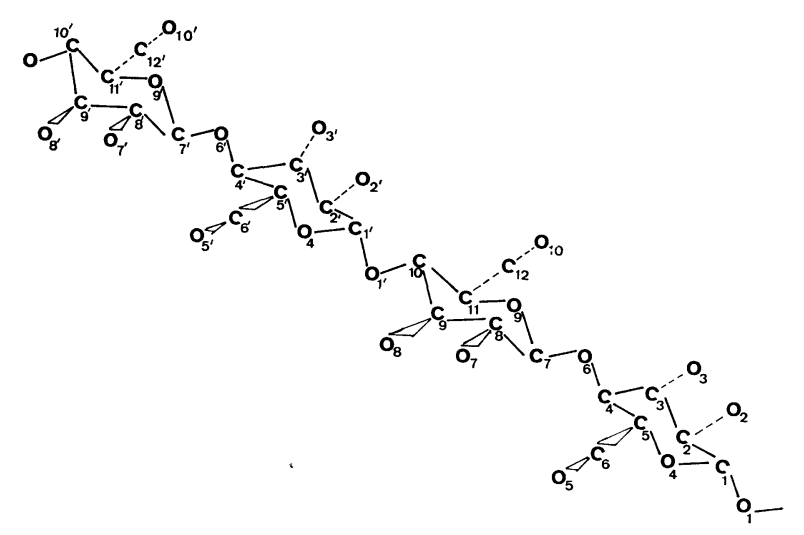

FIG. 5. - Structure d'une molécule de cellulose (notation de Meyer et Misch).

L'hypothèse d'une déformation $\delta(\mathrm{O}-\mathrm{H})$ semble peu probable. En effet, cette bande disparaît après irradiation alors que l'intensité de la bande $v(\mathrm{O}-\mathrm{H})$ augmente. On pourrait imaginer que la déformation $\delta(\mathrm{O}-\mathrm{H})$ cesse sans que soit modifiée l'intensité de la bande $v(\mathrm{O}-\mathrm{H})$; cela est très peu vraisemblable.

$\mathrm{Si}$ certains auteurs, comme Forziati [5] ont pu songer au cycle pyrannosique, c'est qu'ils n'observaient aucune bande d'absorption du cycle. Sans doute est-ce dû au manque de résolution de leur spectrophotomètre, puisque nous avons mis en évidence deux bandes à 948 et $872 \mathrm{~cm}^{-1}$. D'après les indications de Barker [3] sur la cellulose, ces bandes ont une intensité faible : cela explique qu'on ait eu des difficultés à les observer et que nous ayons préféré attribuer la bande à $899 \mathrm{~cm}^{-1}$, qui est assez intense, non pas au cycle mais à un balancement en dehors du plan des atomes d'hydrogène de $\mathrm{C}_{(6)}-\mathrm{H}_{2}$ [7].

L'hypothèse d'une vibration d'allongement $v(\mathrm{C}-\mathrm{O})$ ne peut être écartée puisque la région correspond au domaine habituel des fréquences des vibrations de ce type. En ce sens, on peut retenir l'interprétation de Liang et Marchessault [10] : il se produit une interaction par pont hydrogène entre l'oxygène $O_{(6)}$ et les hydrogènes liés aux deux carbones $\mathrm{C}_{(4)}$ et $\mathrm{C}_{(7)}$ de deux chaînes voisines. Cette interprétation permet de rendre compte effectivement de plusieurs phénomènes :

- Chute de l'indice de polymérisation après irradiation (la bande disparaît totalement).

- Grande sensibilité à l'effet de la deutération [9].

Nous avons conservé la même hypothèse, tout en la complétant : on a bien des vibrations d'allongement $v(\mathrm{C}-\mathrm{O})$; elles sont dues à l'ensemble

$$
\mathrm{C}_{(11)}-\mathrm{O}_{(9)}-\mathrm{C}_{(7)}-\mathrm{O}_{(6)}-\mathrm{C}_{(4)} \text {, }
$$

compte tenu de la présence des deux cycles pyrannosiques qui entourent le pont $\mathrm{C}_{(7)}-\mathrm{O}_{(6)}-\mathrm{C}_{(4)}$. Cette hypothèse est étayée par les trois observations suivantes :

- Dans le domaine $1000-1200 \mathrm{~cm}^{-1}$, on peut observer dans des échantillons de type $S_{16}$ outre la bande à $1162 \mathrm{~cm}^{-1}$ deux bandes à 1036 et $1120 \mathrm{~cm}^{-1}$. Ces bandes ont déjà été signalées dans les spectres de la cellulose [2]. Or, elles sont caractéristiques dans les acétals aliphatiques ou cycliques [11-12], du groupement $\mathrm{C}-\mathrm{O}-\mathrm{C}-\mathrm{O}-\mathrm{C}$ qui apparaît dans la chaîne cellulosique sous la forme

$$
\mathrm{C}_{(11)}-\mathrm{O}_{(9)}-\mathrm{C}_{(7)}-\mathrm{O}_{(6)}-\mathrm{C}_{(4)} \text {. }
$$

- Considérons les ponts $\mathrm{C}-\mathrm{O}-\mathrm{C}$ des composés dont la structure générale est représentée sur la figure 6 .

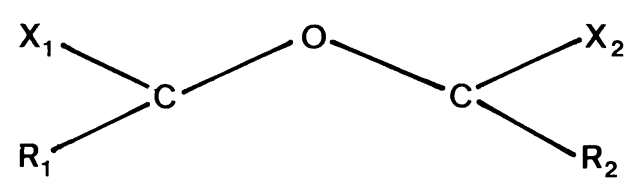

$$
\begin{aligned}
& x_{1}, X_{2} \text { : hydrogène ou oxygène } \\
& R_{1}, R_{2} \text { : groupement quelconque }
\end{aligned}
$$

FIG. 6. - Structure de certains composés possédant un pont $\mathrm{C}-\mathrm{O}-\mathrm{C}$.

On a montré, par des considérations théoriques, que les mouvements de retournement de ces ponts donnent naissance à un groupe de bandes dans la zone $1000-1200 \mathrm{~cm}^{-1}$ [13]. Les fréquences dépendent bien entendu des substituants qui sont ici :

des hydrogènes comme $X_{1}$ et $X_{2}$,

des cycles pyrannosiques comme $\mathbf{R}_{1}$ et $\mathbf{R}_{2}$.

- Enfin ce groupe de bandes se comporte de la même manière vis-à-vis de l'irradiation : il disparaît complètement et laisse place à un fond continu très plat.

5. Spectres d'absorption des films vierges. - 5.1 Films SANS Plastifiant (ÉCHANTILlons S). - L'examen du spectre représenté sur la figure 7 montre que le nombre et l'emplacement des bandes sont ceux des flocons de triacétate de cellulose. Leur forme est nettement plus fine, ce qui peut être dû à l'apparition d'un certain caractère cristallin [19]. L'emploi d'échantillons de plus en plus épais rend le spectre plus diffus ; ce phénomène est particulièrement sensible entre 650 et $200 \mathrm{~cm}^{-1}$; il se produit même une modification du profil des bandes à 475 et $490 \mathrm{~cm}^{-1}$ (Fig. 8). L'identification des bandes au-dessous de $650 \mathrm{~cm}^{-1}$ est difficile, par manque d'informations. Hineo et Yoshinaga [20-21] ont bien publié récemment les spectres du $\beta$-D- glucopyrannose et de la cellobiose entre 500 et $30 \mathrm{~cm}^{-1}$, mais ils ne procèdent à aucune 


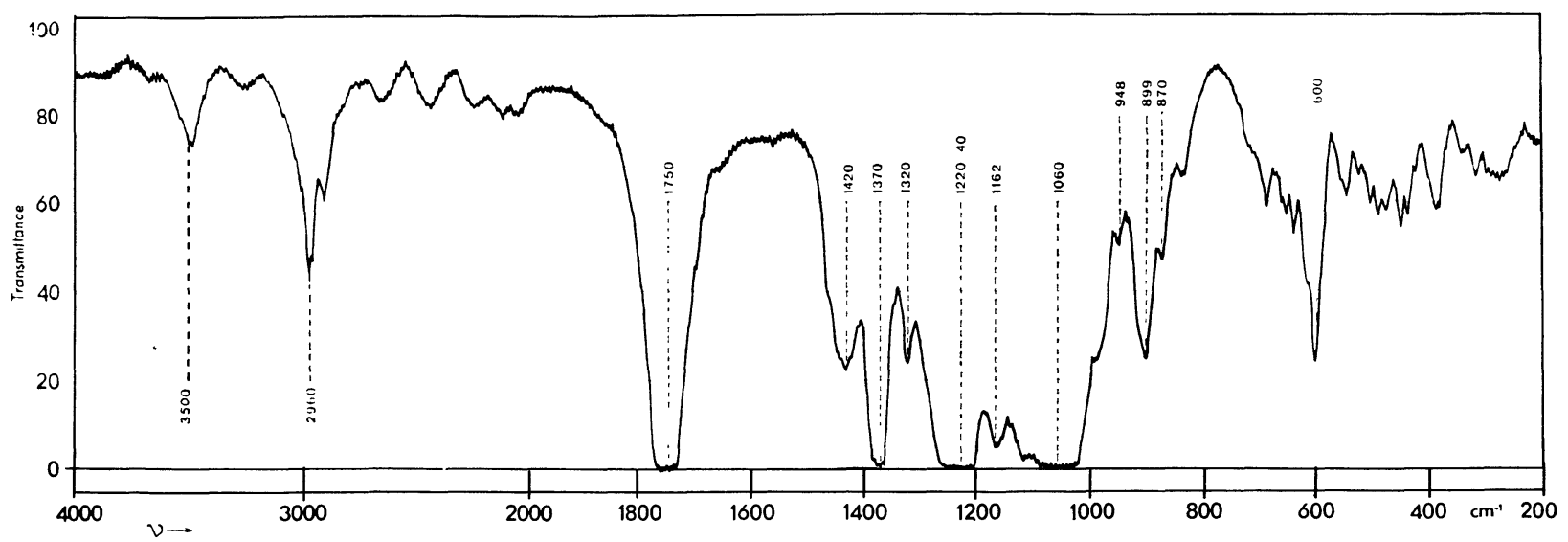

Fig. 7. - Spectre d'absorption d'un film sans plastifiant entre 4000 et $200 \mathrm{~cm}^{-1}$.

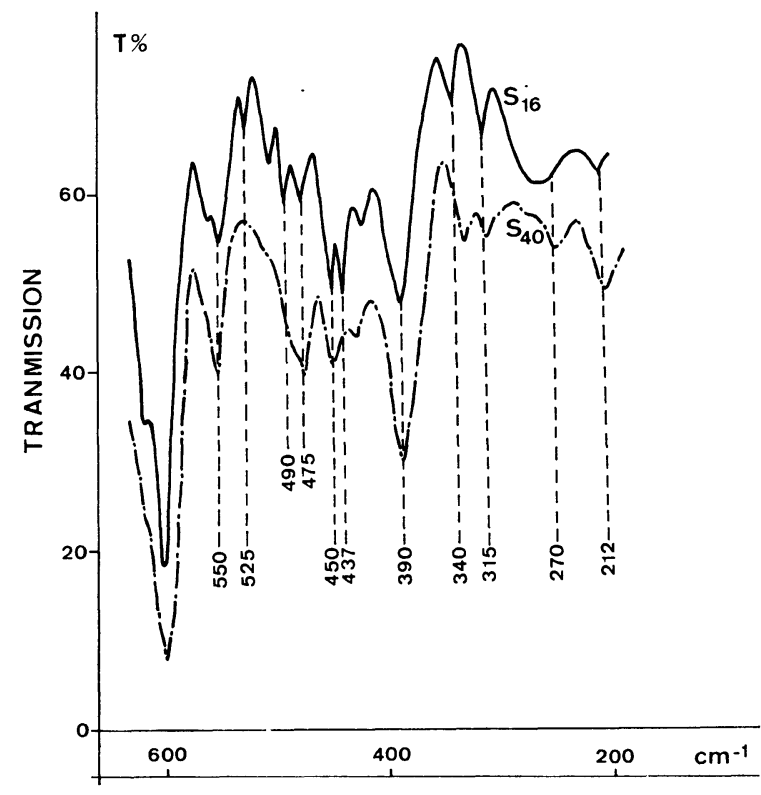

Fig. 8. - Modification du profil des bandes due à un changement d'épaisseur du film.

identification. Le fait le plus caractéristique au-dessous de $200 \mathrm{~cm}^{-1}$ est l'apparition d'une bande à $177 \mathrm{~cm}^{-1}$ (Fig. 9); il s'agit vraisemblablement d'une bande

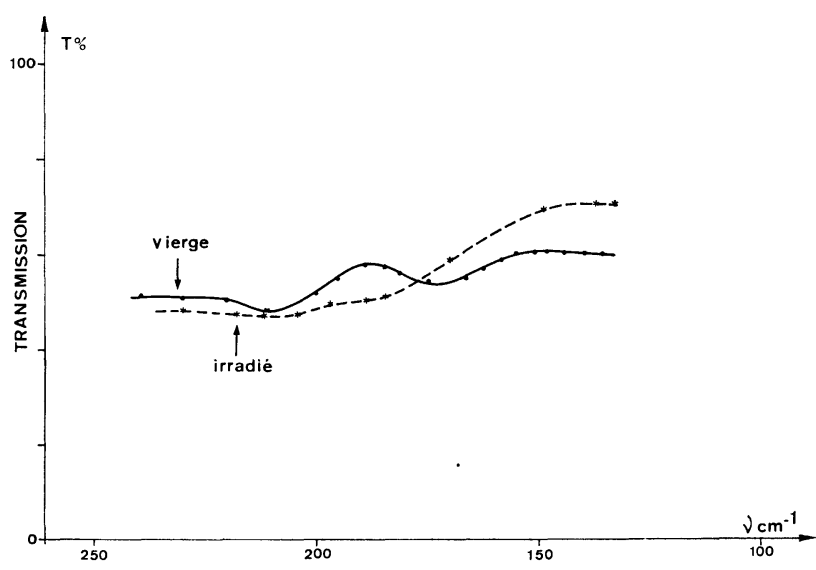

FIG. 9. - Spectre d'absorption d'un film sans plastifiant entre 130 et $200 \mathrm{~cm}^{-1}$

$v(\mathrm{O}-\mathrm{H}-\mathrm{-}-\mathrm{O})$ associée à la formation de liaisons du type pont hydrogène, comme c'est le cas pour d'autres corps comme l'acide acétique, le p-Crésol et l'a-Chlorophénol [22-23].

Au-dessus de $4000 \mathrm{~cm}^{-1}$, nous n'avons trouvé aucune bande d'absorption, du moins jusqu'à $5000 \mathrm{~cm}^{-1}$; le profil est plat aux franges d'interférences d'égale épaisseur près (Fig. 11).

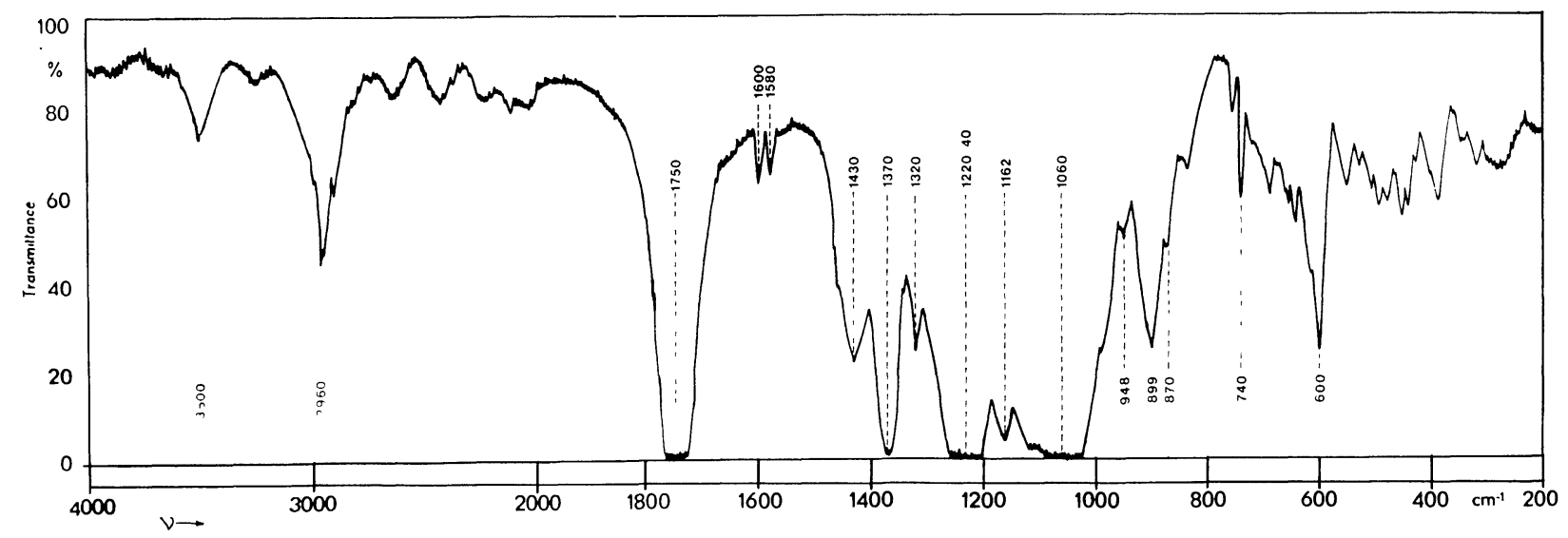

FIG. 10. - Spectre d'absorption d'un film avec plastifiant entre 4000 et $200 \mathrm{~cm}^{-1}$. 


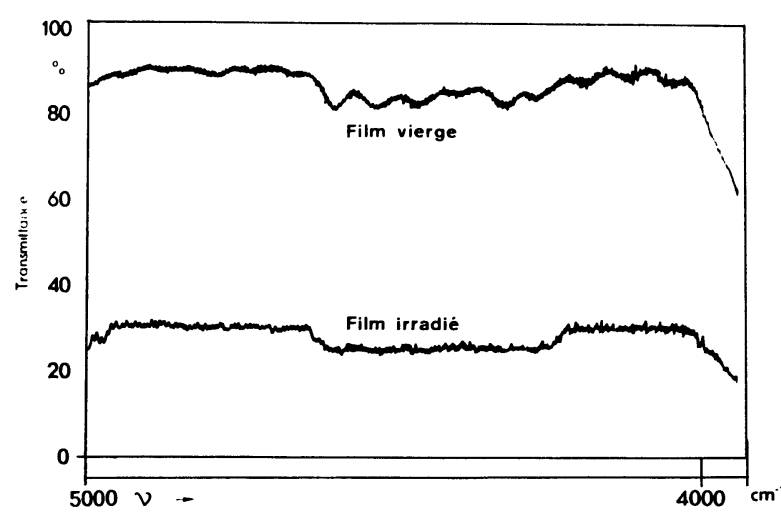

Fig. 11. - Modification de l'intensité relative du fond continu entre 4000 et $5000 \mathrm{~cm}^{-1}$ pour un flux $\Phi$ des ions krypton de $2 \times 10^{12}$ ions $/ \mathrm{cm}^{2}$.

5.2 Films avec plastifiant (échantillons P). - La présence du plastifiant se manifeste par l'apparition dans le spectre précédent de trois nouvelles bandes qui lui sont liées. Elles sont situées à 1600 , $1580,740 \mathrm{~cm}^{-1}$; leurs intensités augmentent en même temps que la teneur en plastifiant (Fig. 10).

6. Modifications apportées aux spectres par l'irradiation. - 6.1 RÉGION $4000-5000 \mathrm{~cm}^{-1}$ (ÉCHANTILLONS $\mathrm{S}$ et P). - L'intensité $I p$ du palier diminue au fur et à mesure que le flux $\Phi$ des particules incidentes croît (Fig. 11). Il est commode d'établir une relation entre $\Phi$ et le rapport $R$ :

$$
R=\frac{(I \mathrm{p}) \text { vierge }}{(I \mathrm{p}) \text { irradié }}
$$

Mais, bien que les intensités soient mesurées avec précision (l'erreur absolue sur (Ip) vierge et (Ip) irradié vaut $1 \%$ de transmission), l'erreur relative commise sur $R$ est importante :

$$
6 \% \text { pour } R=4 \text { et } 25 \% \text { pour } R=20 \text {. }
$$

Les courbes $R(\Phi)$ sont donc surtout représentatives de l'évolution globale des phénomènes. On constate (Fig. 12) que, plus la teneur en plastifiant est élevée, plus le détecteur est sensible aux faibles valeurs de $\Phi$

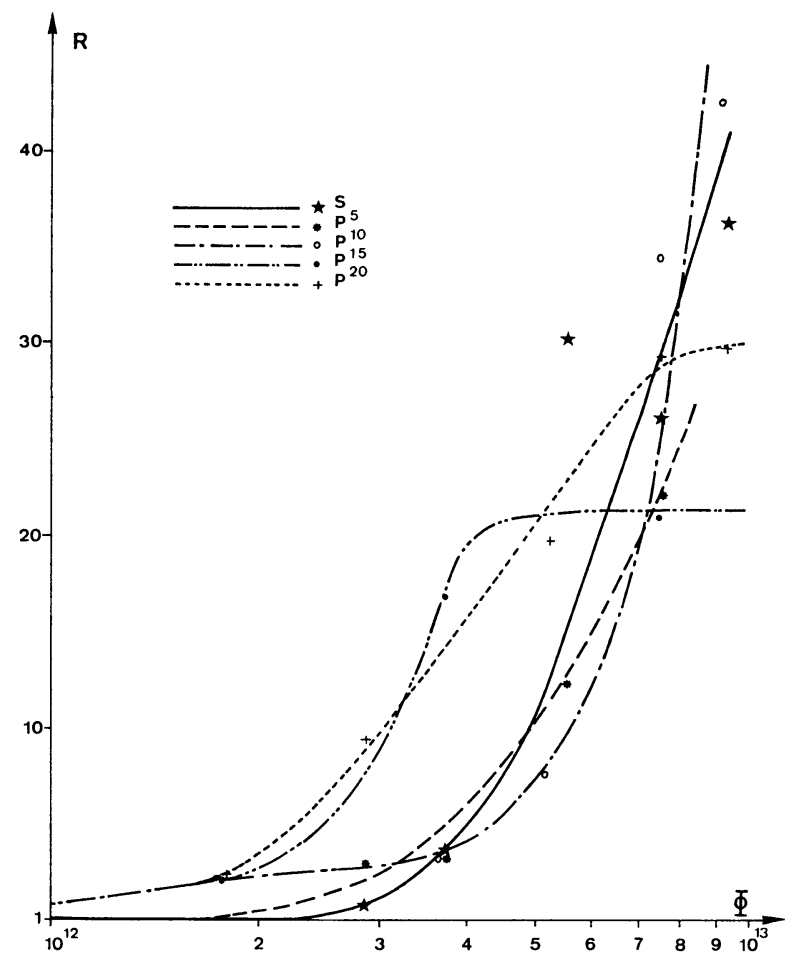

Fig. 12. - Variation de $R(\Phi)$ à $4000 \mathrm{~cm}^{-1}$. ( $\Phi$ en nombre d'ions $/ \mathrm{cm}^{2}$ ).

et plus il a tendance à la saturation pour les plus grandes valeurs de $\Phi$.

6.2 RÉGION 200-130 $\mathrm{cm}^{-1}$ (ÉCHANTILlONS S). La bande située à $177 \mathrm{~cm}^{-1}$ disparaît. D'une manière générale, le spectre a tendance a être moins vallonné.

6.3 RÉGION $4000-200 \mathrm{~cm}^{-1}$. - La figure 13 montre l'effet d'une irradiation de $3,7 \times 10^{12}$ ions krypton $/ \mathrm{cm}^{2}$ sur le spectre d'absorption d'un film sans plastifiant. Le fond continu a augmenté de manière sensible au-dessus de $800 \mathrm{~cm}^{-1}$ mais moins nettement au-dessous.

L'examen du spectre entre 4000 et $800 \mathrm{~cm}^{-1}$, après compensation du faisceau de référence du spectrophotomètre, montre que l'irradiation a tendance à faire disparaître les bandes à 1162 et $948 \mathrm{~cm}^{-1}$

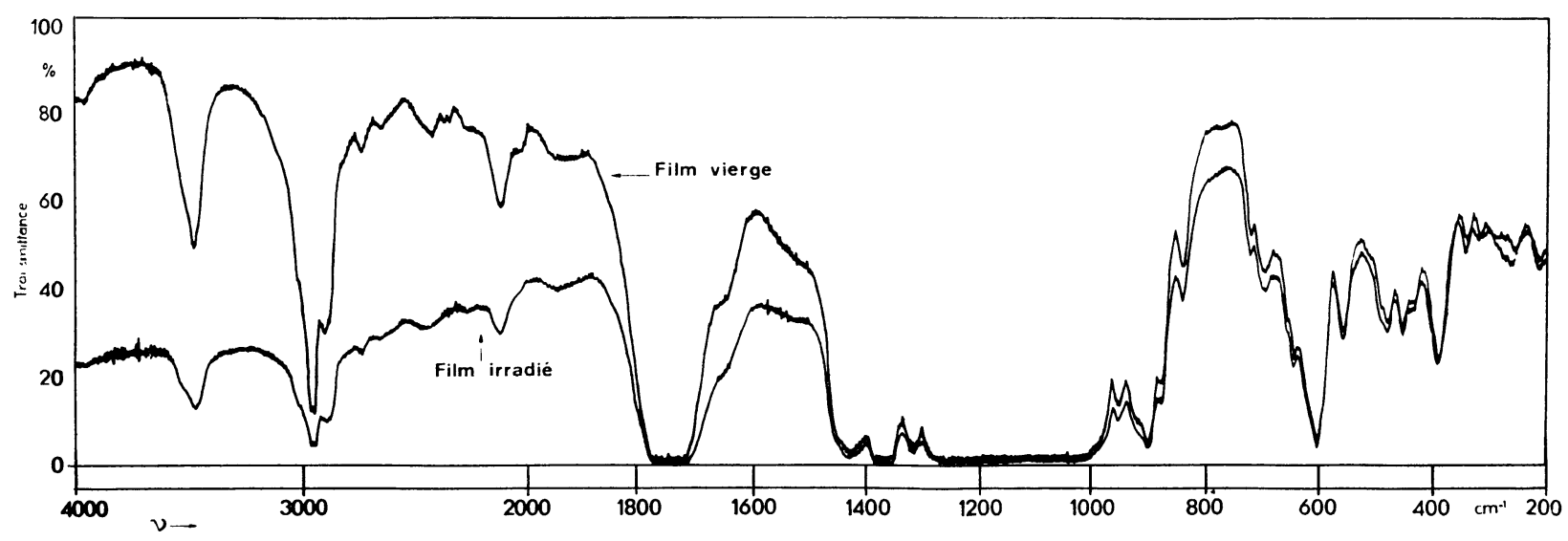

FIG. 13. - Effet d'une irradiation de $3,7 \times 10^{12}$ ions $/ \mathrm{cm}^{2}$ sur un échantillon $\mathrm{S}$. 
et que cette disparition est totale pour un flux de $9,25 \times 10^{12}$ ions $/ \mathrm{cm}^{2}$.

Un examen plus détaillé de la région $600-200 \mathrm{~cm}^{-1}$ (Fig. 14) montre que la bande à $490 \mathrm{~cm}^{-1}$ qui n'apparaissait que sous la forme d'un épaulement de la bande à $475 \mathrm{~cm}^{-1}$ a tendance à se dégager du fond continu au cours de l'irradiation, alors que la bande à $475 \mathrm{~cm}^{-1}$ s'efface progressivement.

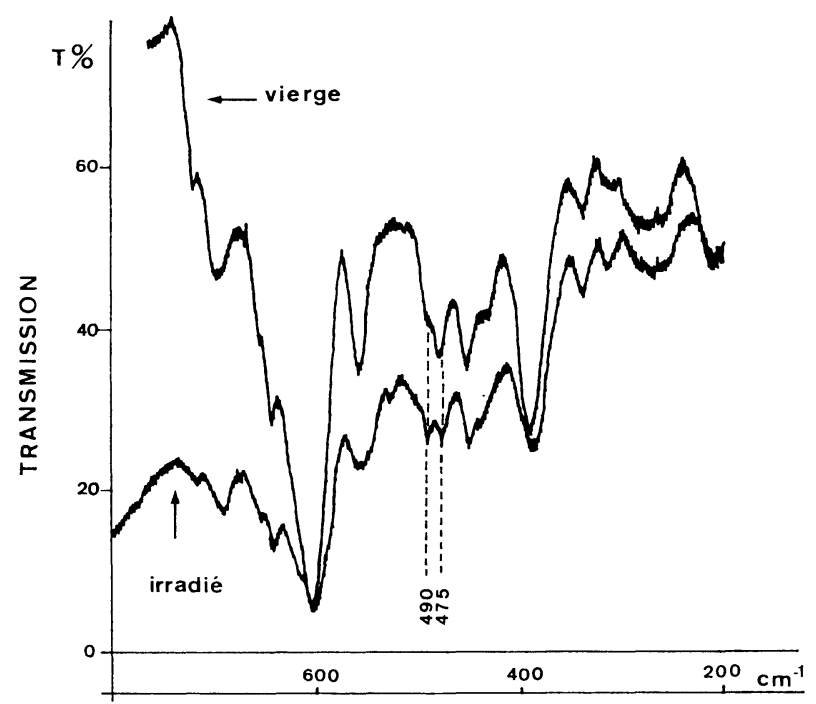

Fig. 14. - Effet d'une irradiation de $9,25 \times 10^{12} \mathrm{ions} / \mathrm{cm}^{2}$ sur les bandes à 475 et $490 \mathrm{~cm}^{-1}$.

Tous ces phénomènes se retrouvent sur les films $P$. De plus, les bandes caractéristiques du plastifiant disparaissent complètement $\left(1580\right.$ et $\left.1600 \mathrm{~cm}^{-1}\right)$ ou sont fortement atténuées $\left(740 \mathrm{~cm}^{-1}\right)$ pour un flux de $9,25 \times 10^{12}$ ions $/ \mathrm{cm}^{2}$ (Fig. 15).

7. Estimation des dommages subis par les films dans le domaine 800-4 $000 \mathrm{~cm}^{-1}$. - Puisque :

$$
\frac{\bar{\rho}}{\rho}=\frac{b}{a} L+\left(1-\frac{b}{a}\right)
$$

la densité volumique $\bar{\rho}$ s'annule non pas pour $L=0$ mais pour :

$$
L_{\mathrm{c}}=\frac{b / a}{(b / a)-1} .
$$

Dans le cas le plus fréquent, nous avons utilisé des échantillons d'épaisseur $b=45 \mu \mathrm{m}$ et des ions krypton de $1,1 \mathrm{MeV}$ par nucléon (soit $a=17 \mu \mathrm{m}$ ). D'où :

$$
L_{\mathrm{c}}=0,627 \text {. }
$$

Il suffit donc que $L$ atteigne la valeur $L_{\mathrm{c}}=0,627$ pour que le groupement chromophore ait totalement disparu dans la zone irradiée. C'est pourquoi dans ce qui suit, les valeurs du flux correspondent toutes à $L=L_{\mathrm{c}}$.

Nous donnons à titre purement indicatif l'allure de la variation de $L(\Phi)$ à $948 \mathrm{~cm}^{-1}$ (Fig. 16). Nous n'en tirons d'ailleurs aucune conclusion d'ordre quantitatif, si ce n'est l'ordre de grandeur de $L_{\mathbf{c}}$.

On peut cependant donner une interprétation, même schématique, de l'irradiation par les ions krypton :

- Un flux de $2 \times 10^{12}$ traces $/ \mathrm{cm}^{2}$ fait disparaître le groupe de bandes de la région $1000-1200 \mathrm{~cm}^{-1}$, c'est-à-dire coupe le groupement $\mathrm{C}_{(7)}-\mathrm{O}_{(6)}-\mathrm{C}_{(4)}$.

- Un flux de $4 \times 10^{12}$ traces $/ \mathrm{cm}^{2}$ provoque la disparition de la bande à $948 \mathrm{~cm}^{-1}$, c'est-à-dire l'ouverture du cycle pyrannosique.

- Un flux de $6 \times 10^{12}$ traces $/ \mathrm{cm}^{2}$ éteint complètement la bande à $899 \mathrm{~cm}^{-1}$, c'est-à-dire détruit les liaisons $\mathrm{C}_{(6)}-\mathrm{H}_{2}$.

Corrélativement, au fur et à mesure que le flux d'ions augmente, les liaisons $\mathrm{C}-\mathrm{H}$ restent pratiquement inchangées tandis que la bande $v(\mathrm{O}-\mathrm{H})$ augmente d'intensité.

En ce qui concerne la liaison $v(\mathrm{C}-\mathrm{O})$ du radical acétyle, la rupture s'effectue à des flux variables selon la teneur en plastifiant du film. Sur les échantillons $\mathrm{P}^{5}$ et $\mathrm{P}^{10}$, elle se produit à $6 \times 10^{12}$ ions $/ \mathrm{cm}^{2}$, tandis que sur les autres échantillons, elle commence à se manifester à partir de $2 \times 10^{12}$ traces $/ \mathrm{cm}^{2}$.

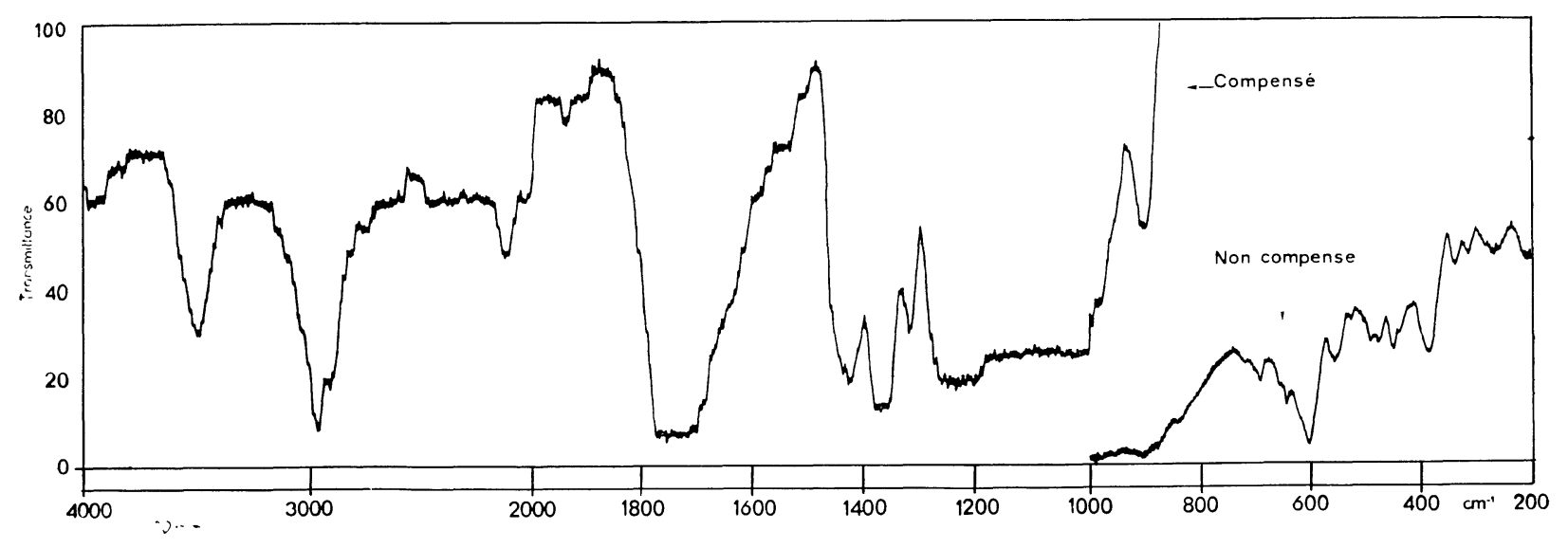

Fig. 15. - Effet d'une irradiation de $9,25 \times 10^{12}$ ions $/ \mathrm{cm}^{2}$ sur un échantillon $P$. 


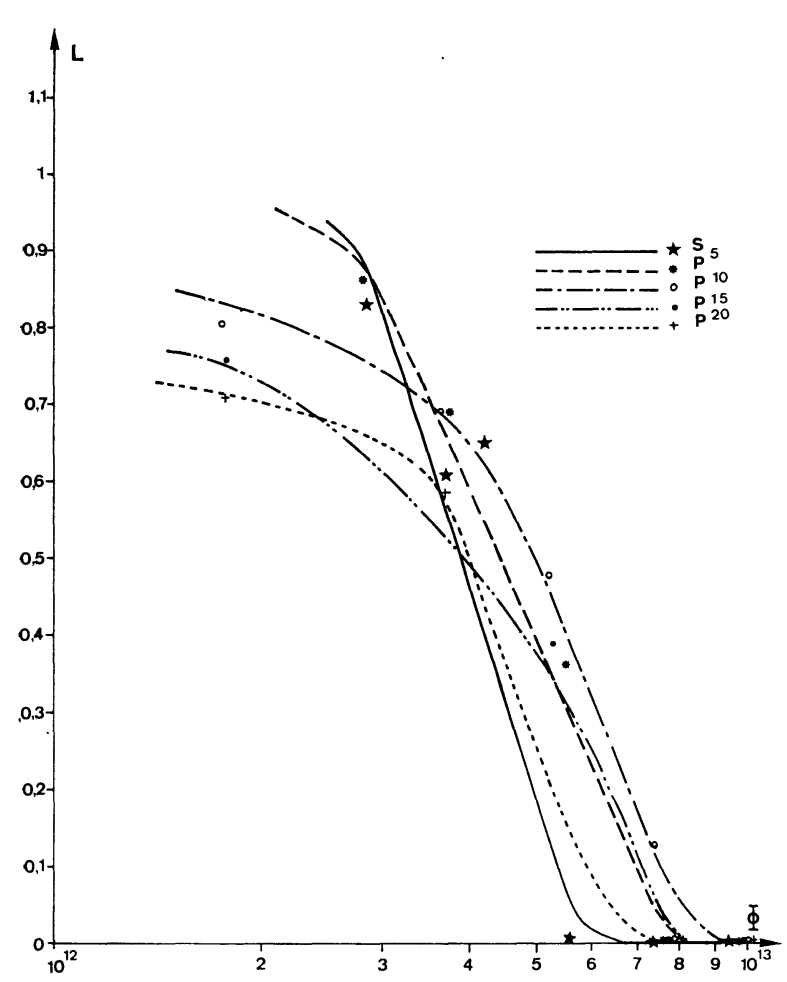

FIG. 16. - Allure de la variation de $L(\Phi)$ à $948 \mathrm{~cm}^{-1}$ $\left(\Phi\right.$ en nombre d'ions $\left./ \mathrm{cm}^{2}\right)$.

L'observation des traces individuelles d'ions krypton confirme ce résultat : dans les échantillons de type $\mathrm{P}^{5}$ et $\mathrm{P}^{10}$, les traces sont nettement mieux définies géométriquement et l'énergie d'activation de l'attaque chimique des films par la potasse est plus élevée.

8. Interprétation des résultats. - L'ouverture du cycle pyrannosique peut être due à la rupture d'une liaison $\mathrm{C}-\mathrm{O}$, soit entre $\mathrm{C}_{(5)}-\mathrm{O}_{(4)}$ soit entre $\mathrm{O}_{(4)}-\mathrm{C}_{(1)}$. Dans ces conditions, l'action destructive des ions krypton se manifesterait surtout au niveau des liaisons $\mathrm{C}-\mathrm{O}$ et, pour les flux les plus élevés, des liaisons $C_{(6)}-H_{(2)}$. C'est l'hypothèse que nous avons retenue.

Pour un flux de $2 \times 10^{12}$ traces $/ \mathrm{cm}^{2}$, les bandes situées entre 1000 et $1200 \mathrm{~cm}^{-1}$ disparaissent, donc les liaisons par les ponts $\mathrm{C}_{(4)}-\mathrm{O}_{(6)}-\mathrm{C}_{(7)}$ entre monomères de $\beta$-D-glucopyrannose ont été détruites.

Une fois ces liaisons détruites, parce que les plus fragiles, ce sont les liaisons $\mathrm{C}-\mathrm{O}$ du cycle pyrannosique qui sont rompues et elles le sont toutes pour un flux de $4 \times 10^{12}$ traces $/ \mathrm{cm}^{2}$, puisque les bandes correspondantes du spectre ont alors disparu.

Enfin, en ce qui concerne au moins les échantillons du type $\mathrm{P}^{5}$ et $\mathrm{P}^{10}$, la rupture de toutes les liaisons $\mathrm{C}-\mathrm{O}$ du radical acétyle exige un flux de $6 \times 10^{12}$ traces $/ \mathrm{cm}^{2}$.

$\mathrm{Au}$ total, pour un flux de $6 \times 10^{12}$ ions $/ \mathrm{cm}^{2}$ et pour des échantillons $\mathrm{P}^{5}$ et $\mathrm{P}^{10}$, il y a destruction de toutes les liaisons $\mathrm{C}-\mathrm{O}$. Comme en moyenne il y a une liaison $\mathrm{C}-\mathrm{O}$ de chaque type par monomère de $\beta$-D-glocopyrannose, on peut dire que les

$$
6 \times 10^{12} \text { ions } / \mathrm{cm}^{2}
$$

ont détruit $3 n$ liaisons $\mathrm{C}-\mathrm{O}$, si $n$ est le nombre total de monomères. La densité des monomères est de $2,926 \times 10^{21}$ puisque la masse molaire est de $288 \mathrm{~g}$ et la masse volumique des films de triacétate est en moyenne de $1,4 \mathrm{~g} / \mathrm{cm}^{3}$. Puisque toutes les liaisons $\mathrm{C}-\mathrm{O}$ sont détruites, tous les monomères ont été attaqués et la totalité du matériau d'épaisseur $a$ a été endommagée. $6 \times 10^{12}$ ions krypton répartis sur une surface de $1 \mathrm{~cm}^{2}$ qui pénètrent dans le matériau sur une profondeur de $17 \mu \mathrm{m}$ endommagent un volume de $17 \times 10^{-3} \mathrm{~cm}^{3}$ soit

$$
\begin{array}{r}
2,926 \times 10^{21} \times \\
\times 17 \times 10^{-3}=4,974 \times 10^{19} \text { monomères ; }
\end{array}
$$

le nombre de liaisons $\mathrm{C}-\mathrm{O}$ détruites est de $3 \times 4,974 \times 10^{19}$. Par conséquent, en moyenne on peut estimer qu'un ion krypton a détruit :

$$
\frac{3 \times 4,974 \times 10^{19}}{6 \times 10^{12}}=2,48 \times 10^{7} \text { liaisons } \mathrm{C}-\mathrm{O} \text {. }
$$

On peut retrouver ce résultat à partir de considérations purement énergétiques. Sachant que l'énergie moyenne de dissociation d'une liaison $\mathrm{C}-\mathrm{O}$ est de $85 \mathrm{kc} /$ mole [24] et que l'énergie des ions krypton est de $1,1 \mathrm{MeV} /$ nucléon (soit 3,52 $\times 10^{-12}$ cal.), le nombre de liaisons C-O rompues par un ion est de $2,49 \times 10^{7}$.

9. - Spectrométrie X. - Nous avons effectué une tentative de spectrométrie $X$ par la méthode de Bragg à l'aide d'un appareil Cristalobloc C. G. R., modèle 31, muni d'un monochromateur. Les spectres étant assez diffus, il a fallu utiliser de courtes longueurs d'onde : nous nous sommes servis de la raie $\mathrm{K} \alpha$ du cuivre.

Quel que soit le type de film, les spectres des échantillons vierges présentent tous deux maxima à $4,6^{\circ}$ et $9^{\circ}$ (Fig. 17). Les positions et les intensités relatives

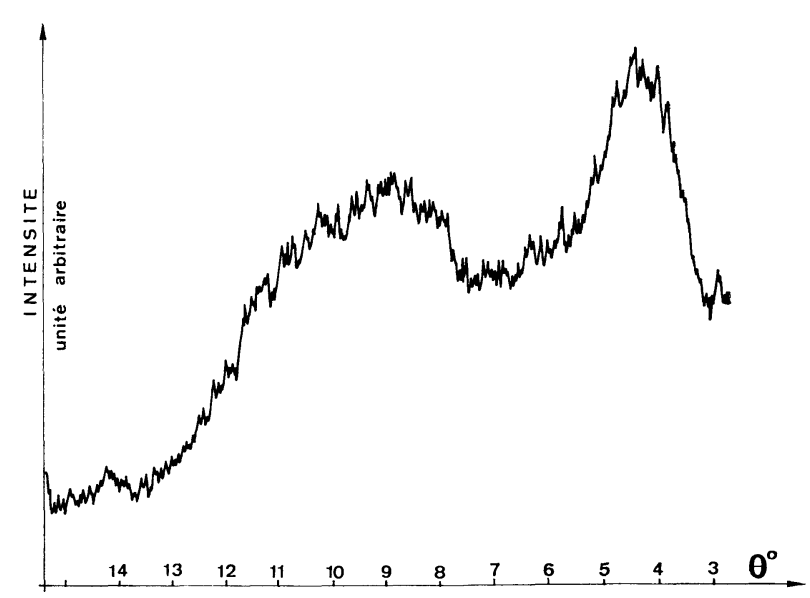

Fig. 17. - Spectre de rayons $\mathrm{X}$ d'un échantillon vierge.

de ces deux maxima sont en bon accord avec les valeurs fournies par la littérature [26].

Les effets de l'irradiation sont quasi nuls. On ne note aucune variation de l'intensité du fond continu 
et des deux maxima, ni aucun déplacement de ces deux maxima. L'irradiation ne provoque donc aucune modification sensible de structure; les produits des réactions chimiques dues à l'action des ions restent localisés sur les mêmes sites.

10. Conclusion générale. - La présente analyse a montré le rôle prépondérant joué par les liaisons $\mathrm{C}-\mathrm{O}$ dans le processus d'interaction des ions lourds et du triacétate de cellulose à l'air libre.

Elle pose aussi un certain nombre de questions :

- Plusieurs interprétations [27-28-29] reposent sur un mécanisme radicalaire induit par des peroxydes. Nous n'avons pas décelé de bandes d'absorption de ces peroxydes. Il est vrai qu'elles doivent avoir des intensités faibles et se trouver entre 820 et $1000 \mathrm{~cm}^{-1}$, c'est-à-dire dans une région où le fond continu est important.

- De nombreuses études faites par R. P. E. [24-29-30] on mis en évidence l'existence de radicaux libres de longue durée de vie. Ces radicaux seraient localisés sur les carbones [1] et [5] ou [6] du monomère de $\beta$-D-glucopyrannose. L'existence de ces radicaux est compatible avec nos résultats qui mettent en évidence la rupture des liaisons $\mathrm{C}_{(4)}-\mathrm{O}_{(6)}-\mathrm{C}_{(7)}$; $\mathrm{C}_{(6)}-\mathrm{O}_{(5)}$ (radical acétyle) et l'ouverture du cycle pyrannosique par $\mathrm{C}_{(5)}-\mathrm{O}_{(4)}-\mathrm{C}_{(1)}$. Par contre, nos expériences, qui sont effectuées plusieurs semaines après l'irradiation, montrent que ces radicaux libres ne sont pas stables ou bien qu'ils ne sont pas liés à la présence de peroxydes.

D'après Sears et Bopp [31], les gaz produits par une irradiation $\gamma$ ou $\alpha$ de cellulose, de diacétate, de triacétate et de nitrate de cellulose sont :

$\mathrm{CO}, \mathrm{CO}_{2}, \mathrm{H}_{2}$ pour tous ces composés;

$\mathrm{CH}_{4}$ pour les acétates et le nitrate ;

$\mathrm{NO}_{2}$ pour le nitrate.

La décarboxylation observée s'explique à partir des ruptures des liaisons $\mathrm{C}-\mathrm{O}$ que nous avons signalées. Reste le cas du méthane pour le nitrate de cellu- lose ; la seule possibilité est que le nitrate contienne un plastifiant qui possède le radical acétyle, ce que ne précisent pas Sears et Bopp.

La présence d'un radical de longue durée de vie repose sur une forte délocalisation de l'électron célibataire qui ne peut se produire que si l'environnement immédiat de cet électron est riche en électrons $\pi$. Parallèlement à la formation du radical, devraient donc apparaître des liaisons $\mathrm{C}=\mathrm{C}$ ou des hybridations d'orbitales par des cycles contenant 4, 5 ou 6 atomes de carbone. L'élargissement vers les basses fréquences, lors de l'irradiation, de la bande $v(\mathrm{C}=\mathrm{O})$ à $1750 \mathrm{~cm}^{-1}$, peut être interprétée par la formation d'une liaison $\mathrm{C}=\mathrm{C}$, bien que normalement la présence de l'ion

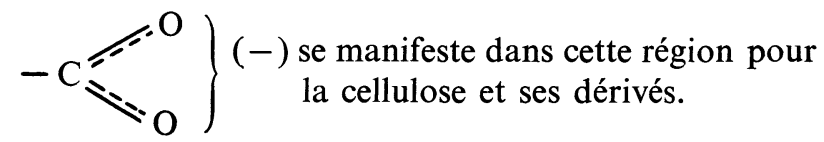

Enfin, les travaux de Rozmarin et Butnaru [32] ont montré la formation du cycle furannique, ce que ne laisse pas prévoir la spectrographie infrarouge. Il est vrai qu'il s'agit d'irradiations par des rayons gamma.

Quoi qu'il en soit, la détérioration du matériau par les ions lourds explique que les zones irradiées aient un comportement spécifique vis-à-vis d'une attaque chimique. Celle-ci provoque un rongement général du film détecteur et en même temps se développe de façon préférentielle dans les régions traversées par les ions lourds. Dans certaines conditions l'attaque chimique permet d'obtenir des traces observables au microscope optique. Ces traces visualisent alors le passage des ions dans le matériau.

Remerciements. - Nos remerciements vont à M. Gerbier avec lequel nous avons eu de fructueuses discussions et d'une manière générale au Laboratoire de Physique Moléculaire de notre Université qui nous a permis de réaliser les expériences de spectrographie infrarouge. Nous tenons également à remercier le laboratoire de Chimie Minérale de notre Université où a été effectuée la spectrométrie $X$.

\section{Bibliographie}

[1] Quintard, P. et Delorme, P., Revue Phys. Appl. 2 (1967) 225.

[2] NAKANISHI, K., Infrared Absorption Spectroscopy (Molden. Day Inc., San Francisco) 1969.

[3] Barker, S. A., Bourne, E. J., Stacay, M. et Whiffen, D. M., J. Chem. Soc. (1954) 171.

[4] Baggett, N., Barker, S. A., Foster, A. B., Moore, R. H. et Whiffen, D., J. Chem. Soc. (1960) 4565.

[5] Forziati, F. H. et Rowen, J. W., N. B. S. 46 (1951) 38.

[6] Durie, R. A., Lynch, B. M. et Sternheil, S., J. Chem. Soc. (1960) 3575.

[7] O'Connor, R. T., The encyclopedia of Spectroscopy (Reinhold Publ. Corp.), 1960.
[8] Segal, L., O'Connor, R. P. et Egerton, F. V., J. Ann. Chem. Soc. 82 (1960) 2807.

[9] Higgins, H. G., Stewart, C. M. et Harrington, K. J., $J$. Polymer. Sci. 51 (1961) 59.

[10] Liang, C. Y. et Marchessault, R. H., J. Polymer. Sci. 37 (1959) 385.

[11] Bergmann, E. D. et Pinchas, S., Rev. Trav. Chem. 71 (1952) 161

[12] Tsubor, M., J. Polymer. Sci. 25 (1957) 159.

[13] KonarsKi, J., J. Mol. Struct. 7 (1971) 19.

[14] Liang, C. Y. et Marchessault, R. H., J. Polymer Sci. 39 (1959) 269. 
[15] O’Connor, R. T., Dupre, E. F. et McCall, E. R., Analytical Chemistry 29 (1957) 998.

[16] O’Connor, R. T., Dupre, E. F. et McCall, E. R., Textil Research J. 28 (1958) 542.

[17] Brown, L., Holliday, P. et Trotter, I. F., J. Chem. Soc. (1951) 1532.

[18] Durie, R. A. et Sternhell, S., Austr. J. Chem. 12 (1959) 205.

[19] White, J. W., Eddy, C. R., Petty, J. et Hoban, N., Analytical Chemistry 30 (1958) 506.

[20] Hineo, M. et Yoshinaga, H., Spectrochimica Acta 28A (1972) 2263.

[21] Hineo, M. et Yoshinaga, H., Spectrochimica Acta 29A (1973) 1575.

[22] Hurley, W. J., Kuntz, I. D. et Leroi, G. E., J. Ann. Chem. Soc. 88 (1966) 3199.

[23] Moller, K. D. et Rothschild, W. G., Far Infrared Spectroscopy (Wiley Interscience, New York) 1971.
[24] Phillip, G. O., Hinojosa, O., Arthur, J. C. et Mures T., Textile Research J. 36(1966) 822.

[25] Vareille, J. C. et Teyssier, J. L., Radioprotection 7 (1972) 215.

[26] Buntuakov, A. S. et Averyanova, M., J. Polymer. Sci. 38 C (1972) 109.

[27] Marchand, S. et Puig, J. P., Conf. Polymères Greffes (Mulhouse) 1972.

[28] Monnin, M. et Blanford G., C. R. Hebd. Séan. Acad. Sci. 276 (1973) 399.

[29] Bangh, P. J., Hinojosa, O. et Arthur, J. C., J. Appl. Polymer. Sci. 11 (1967) 1139.

[30] Deffner, U. et Paretzke, H., Rad. Res. 49 (1972) 272.

[31] Sears, D. et Bopp, D. O., Rapport O. R. N. L. (1968) 3151.

[32] Rozmarin, G. H. et Butnaru, R., Cellulose Chem. and Techn. 6 (1972) 393.

[33] Avan, L., Avan, M., Blanc, D. et Teyssier, J. L., Ionographie (Doin Paris) 1973. 